\title{
Correction to: Differentiation of Malignant and Benign Head and Neck Tumors with Amide Proton Transfer-Weighted MR Imaging
}

Lu Yu, ${ }^{1,2}$ Chunmei Li, ${ }^{1}$ Xiaojie Luo, ${ }^{1}$ Jinyuan Zhou, ${ }^{3}$ Chen Zhang, ${ }^{1}$ Yi Zhang, ${ }^{4}$ Min Chen ${ }^{1,2}$

${ }^{1}$ Department of Radiology, National Center of Gerontology, Beijing Hospital, No. 1 Da-Hua Road, Dong Dan, Beijing, 100730, China

${ }^{2}$ Graduate School of Peking Union Medical College, No. 9 Dong Dan San Tiao, Beijing, 100730, China

${ }^{3}$ Department of Radiology, Johns Hopkins University, 600 N. Wolfe Street, Park 336, Baltimore, MD, 21287, USA

${ }^{4}$ Center for Brain Imaging Science and Technology, Key Laboratory for Biomedical Engineering of Ministry of Education, College of Biomedical Engineering \& Instrument Science, Zhejiang University, No. 388 Yuhangtang Road, Zhejiang, 310058, Hangzhou, China

Correction to: Mol Imaging Biol (2019) 21:348Y355.

https://doi.org/10.1007/s11307-018-1248-1

This article is about head and neck tumor MR imaging and is not within the scope of two National Institutes of Health grants (R0ICA166171, ROICA228188) erroneously listed. Thus, the authors would like to remove the reference to the NIH grants R01CA166171 and R01CA228188 in this article.

The authors regret this error.

Publisher's Note Springer Nature remains neutral with regard to jurisdictional claims in published maps and institutional affiliations.

The online version of the original article can be found at https://oi.org/ $10.1007 / \mathrm{s} 11307-018-1248-1$

Correspondence to: Min Chen; e-mail: cjr.chenmin@vip.163.com 